\title{
O trabalho interprofissional nas visitas domiciliares do PET-saúde: um relato de experiência
}

\author{
Interprofessional work in PET-health home visits: an experience report
}

Trabajo interprofesional en visitas a domicilio de PET-salud: un informe de experiencia

Tereza Cristina dos Reis Ferreira ${ }^{1 *}$, Erica Feio Carneiro Nunes ${ }^{1}$, Nilson Veloso Bezerra ${ }^{1}$, Ana Carolina de Souza Lopes ${ }^{1}$, Andreya Araújo Gomes ${ }^{1}$, Anne Beatriz Duarte da Conceição ${ }^{1}$, Bárbara Corrêa Moura ${ }^{1}$, Deizyane dos Reis Galhardoํ․ Gabriel Paz de Lima ${ }^{1}$, Willame Oliveira Ribeiro Junior ${ }^{1}$.

\section{RESUMO}

Objetivo: Relatar a experiência de graduandos em saúde de uma universidade estadual, participantes do Programa de Educação pelo Trabalho para a Saúde - PET-Saúde/Interprofissionalidade, em visitas domiciliares a idosos atendidos pela equipe. Relato de experiência: Realizou-se visitas semanais com 5 idosos durante dois meses de 2019, além da aplicação de escalas de avaliação e o preenchimento da caderneta do idoso e pode-se observar: a falta de apoio familiar, fatores de riscos, negligência quanto a alimentação ou uso de medicamentos, entre outras situações. Ressalta-se que apesar das limitações, a diversidade de áreas do grupo permitiu uma visão conjunta e interprofissional, analisando aspectos essenciais para o trabalho envolvendo o idoso em seu aspecto biopsicossocial e possibilitando a construção do Projeto Terapêutico Singular (PTS). Considerações finais: As visitas domiciliares são uma estratégia para o acompanhamento do idoso em vários âmbitos, além de contribuir para a formação acadêmica e profissional, desenvolvendo competências voltadas para o atendimento humanizado.

Palavras-chave: Visita domiciliar, Idoso, Atenção primária à saúde.

\section{ABSTRACT}

Objective: Report experience of undergraduate health students at a state university, participants of Health Work Education Program - WEP-Health/Interprofessionality, in home visits to the elderly attended by the health team. Experience report: Weekly visits has been carried out with 5 elderly people during two months of 2019, in addition to the application of assessment scales and the filling out of the elderly person's handbook and it can be observed: lack of family support, risk factors, neglect regarding food or use medication, among other situations. It is noteworthy that despite the limitations, the diversity of areas of the group allowed a joint and interprofessional view, analyzing essential aspects for the work involving the elderly in their biopsychosocial aspect and enabling the construction of the Singular Therapeutic Project (STP). Final considerations: Home visits are a strategy for monitoring the elderly in various areas, in addition to contributing to academic and professional training, developing skills aimed at humanized care.

Keywords: House calls, Aged, Primary health care.

\section{RESUMEN}

Objetivo: Informar sobre la experiencia de estudiantes de pregrado en salud en una universidad estatal, participando en el Programa de Educación a través del Trabajo para la Salud - PET-Saúde / Interprofesionalidad, en visitas domiciliarias a los ancianos atendidos por el equipo. Informe de experiencia: Se realizaron visitas semanales a 5 personas mayores durante dos meses de 2019, además de la aplicación de escalas de evaluación y el llenado del manual de la persona mayor, y se puede observar lo siguiente: falta de apoyo familiar, factores de riesgo, negligencia con respecto a alimentación o uso de medicamentos, entre otras situaciones. Es de destacar que a pesar de las limitaciones, la diversidad de áreas del grupo permitió una visión conjunta e interprofesional, analizando aspectos esenciales para el trabajo que involucra a los ancianos en su aspecto biopsicosocial y permitió la construcción del Proyecto Terapéutico Singular (PTS). Consideraciones finales: Las visitas domiciliarias son una estrategia para monitorear a los ancianos en diversas áreas, además de contribuir a la capacitación académica y profesional, desarrollando habilidades dirigidas a la atención humanizada.

Palabras clave: Visita domiciliaria, Anciano, Atención primaria de salud.

1 Universidade do Estado do Pará (UEPA), Belém - PA. *E-mail: reis.tereza@gmail.com SUBMETIDO EM: 6/2020 | ACEITO EM: 7/2020 | PUBLICADO EM: 9/2020 


\section{INTRODUÇÃO}

Historicamente, o Brasil se apresenta como sendo um país jovem, visto que, a maior parte de sua população, de acordo com o SENSO de 2010 do Instituto Brasileiro de Geografia Estatística (IBGE) se apresentava na faixa etária de até 24 anos de idade, cenário que se modifica cada vez mais. Atualmente $9,83 \%$ da população brasileira possui mais de 65 anos de idade e estima-se que no ano de $206025,49 \%$ dos habitantes serão idosos (IBGE, 2020).

Isso demonstra que a cada dia ocorrem alterações na pirâmide etária brasileira, a qual possui um número significativo de idosos que tende a aumentar nos próximos anos. Dessa forma, tal transição demográfica traz à tona a discussão acerca da implementação e efetividade de políticas públicas que visem garantir direitos básicos da população idosa, tais como a assistência à saúde, fortalecendo o envelhecimento saudável (MIRANDA GDM, et al., 2016).

Dentre as notáveis questões referentes ao envelhecimento e a saúde, principalmente na atenção primária, não se pode deixar espaços para a falta de planejamento por parte dos órgãos públicos. Uma vez que, a partir dos 50 anos de idade, principalmente, o autocuidado e a mudança de hábitos são importantíssimos para a melhoria da qualidade de vida, e tais ações de conscientização devem ser prestadas e ensinadas pelos profissionais atuantes ao seu público alvo. Uma das principais formas para a promoção da saúde a pessoas idosa se concentra em visitas domiciliares (CASTRO APR, et al., 2018).

Através da portaria $N^{\circ} 2.488$, de 21 de Outubro de 2011, há a aprovação de políticas de atenção básica, como as visitas domiciliares (BRASIL, 2011). Desde então podem ser realizadas, através do Agente Comunitário de Saúde (ACS), que é um dos membros da Estratégia Saúde da Família (ESF) e uma equipe composta por profissionais de diversas áreas como, médico, enfermeiro e biomédico (ANDRADE VMP e CARDOSO CL, 2017).

Neste contexto, destacamos o Programa de Educação pelo Trabalho para a Saúde (PET-Saúde) como um possível contribuidor no que tange às visitas domiciliares. O PET Saúde foi instituído no ano de 2010, por meio das Portarias GM/MS no 421 e 422, oriundo de uma iniciativa do Ministério da Saúde com o objetivo de qualificação dos profissionais de saúde em conjunto com a formação dos acadêmicos de graduação de saúde. O Programa se utiliza da educação pelo trabalho, voltado para as ações de ensino-serviço-comunidade, dessa forma envolvendo ações de ensino, pesquisa, extensão e a participação social (BRASIL, 2020).

Em julho de 2018, foi publicado o edital da edição "PET - Saúde Interprofissionalidade", com os preceitos do projeto junto a Educação Interprofissional (EIP). A EIP ocorre quando dois ou mais profissionais ou acadêmicos aprendem entre si, com e sobre as outras atuações profissionais, com objetivo de melhoria, colaboração e qualidade nos atendimentos e serviços prestados, segundo o Centro para Aprovação da Educação Interprofissional (CAIPE) (REIP, 2018).

Dessa forma, este estudo tem como objetivo relatar a experiência de acadêmicos de graduação em saúde de uma universidade estadual que participam do PET-Saúde Interprofissionalidade nas visitas domiciliares a idosos atendidos pela equipe.

\section{RELATO DE EXPERIÊNCIA}

Trata-se de um relato de experiência vivenciado por acadêmicos da Universidade do Estado do Pará, inseridos no Programa de Educação pelo Trabalho para a Saúde - PET-Saúde/Interprofissionalidade em parceria com a Secretaria Municipal de Saúde de Belém. A vivência aconteceu durante o mês de outubro e novembro de 2019, no qual foram realizadas oito visitas domiciliares a cinco idosos usuários do SUS, que residem próximo à Unidade Municipal de Saúde (UMS), de um bairro localizado no município de Belém do Pará.

As visitas eram realizadas por subgrupos, a fim de melhor atender as demandas de cada usuário e evitar possíveis barreiras, uma vez que o grupo geral é composto por onze acadêmicos e três preceptoras (profissionais da unidade). A divisão era realizada de acordo com as escalas de cada participante e com a 
disponibilidade dos usuários, dessa forma, cerca de três acadêmicos de cursos diferenciados e duas preceptoras realizavam as visitas, acompanhados, ou não, de um agente comunitário de saúde (ACS).

Todos os idosos foram avaliados por meio de uma entrevista semiestruturada e por meio da aplicação de instrumentos validados, tais como a Escala de Depressão Geriátrica (GDS), Mini exame do Estado Mental (MEEM) e escala de vulnerabilidade Vulnerable Elders Survey-13 (VES-13). Por fim, realizava-se o cadastro ou atualização da caderneta do idoso e "feedback" da avaliação ao usuário e/ou seu (s) familiar (es) ou cuidador(es).

Nas visitas realizadas pôde-se observar que os cinco idosos avaliados apresentavam limitações consideráveis, desde condições biológicas e físicas, quanto questões sociais. A maioria residia em casas pequenas, insalubres, com pouca higienização e iluminação. Durante a experiência, notou-se grande dificuldade dos mesmos na realização de algumas necessidades como, por exemplo, acessibilidade nos diferentes locais da casa, sobretudo, banheiros e quartos, uma vez que o acesso a esses compartimentos, geralmente, era dificultado por barreiras físicas ou estruturais. Além disso, na maioria dos casos pôde-se notar a falta de apoio familiar, apesar de todos relatarem possuir familiares próximos ou residirem com os mesmos.

Outro aspecto observado na maior parte das residências, foi a constante presença fatores de riscos de quedas, tais como objetos espalhados pelo chão, exacerbação de tapetes, animais domésticos, pedras, lixo, entre outros. Outrossim, também foi possível identificar que alguns idosos sofriam negligência quanto a sua alimentação, ficando muitas horas sem comer e com uma ingesta hídrica inadequada. Com relação a alimentação também foi percebido, em algumas casas, a grande quantidade de insumos industrializados, assim como, o horário desregulado para a alimentação.

Outra questão observada, trata-se da capacidade de ir e vir para determinados lugares, tais como a unidade de saúde, a qual, apesar de encontrar-se próxima a residência destes idosos, os mesmos pouco à frequentavam, devido a questões relacionadas à dificuldade de locomoção ou a falta de um acompanhante para levá-los. Tal situação pode contribuir para o surgimento e/ou agravamento de condições de saúde, que poderiam ser evitadas ou tratadas sem gerar grandes consequências.

Da mesma maneira, verificou-se que os idosos também são negligenciados quanto ao uso de medicamentos. Todos apresentavam alguma condição clínica como Diabetes Mellitus, Hipertensão e/ou problemas pulmonares que precisa ser tratada com medicamento de maneira correta. Entretanto, em nenhum dos cinco casos foi possível presenciar a realização desta tarefa. Os idosos faziam uso dos medicamentos em horários irregulares, por vezes deixavam de tomar por esquecimento ou por falta da medicação adequada.

A falta do remédio foi questionada e, em algumas das situações, foi relatado que o mesmo não havia sido comprado ou que o familiar responsável não havia solicitado no posto de saúde, o qual, fornece os remédios de maneira gratuita. Posteriormente a visita, tais pontos observados eram discutidos com o grupo geral, a fim de traçar o Projeto Terapêutico Singular (PTS) mais adequado a cada idoso.

\section{DISCUSSÃO}

O envelhecimento populacional é um dos maiores desafios da saúde pública contemporânea, sobretudo nos países em desenvolvimento. Apesar do grande quantitativo de idosos no mundo, este público, em sua maioria, é exposto a péssimas condições de vida, os quais, muitas vezes, apresentam-se em situações de abandono, tanto por seus familiares, quanto pela própria sociedade (MIRANDA GMD, et al., 2016).

No Brasil, o Estatuto do Idoso, criado por meio da Lei $n^{\circ} 10.741$ de $1^{\circ}$ de outubro de 2003 , visa valorizar o idoso como cidadão e ampará-lo nesta etapa crucial da vida. Por meio desta lei, estabeleceu-se que o Estado e a família precisam garantir a dignidade da pessoa idosa, assim como, a proteção contra violências e/ou abuso, sejam estas de ordem física, psicológica ou social (BRASIL, 2007).

Para o desenvolvimento mais eficaz do trabalho, foi criada uma entrevista semiestruturada, contendo informações fundamentais para a avaliação do idoso e seu contexto de vida. Além disso, era realizado a aplicação da Escala de Depressão Geriátrica (GDS), caracterizada por um instrumento com quinze perguntas, sendo as respostas objetivas de "sim/não" a respeito de como o indivíduo se sente, logo, era identificado 
possíveis estados depressivos. De acordo com a tabela de pontuação, caso o indivíduo obtivesse o escore entre onze a quinze, era sinalizado um quadro de depressão severa, por exemplo (PARADELA EMP, et al 2005).

Além disso, com a finalidade de avaliar o estado mental dos idosos domiciliados aplicou-se o Mini Exame do Estado Mental (MEEM). Este instrumento foi projetado para ser uma avaliação clínica prática de mudança do estado cognitivo em pacientes geriátricos, examinando a orientação temporal e espacial, memória de curto prazo (imediata ou atenção) e evocação, cálculo e habilidades de linguagem e viso-espaciais, considerando notas de corte para déficit cognitivo 18/19 para pessoas analfabetas e 24/25 para os escolarizados, adaptadas para a sociedade brasileira (LOURENÇO RA e VERAS RP, 2006; MELO DM e BARBOSA AJG, 2015).

Com intuito de ampliar e integrar a atividade interprofissional da equipe, o Plano Terapêutico Singular (PTS) foi construído para essa população alvo a fim de atender os usuários vulneráveis. Esses idosos eram triados a partir da escala de vulnerabilidade Vulnerable Elders Survey-13 (VES-13) e da demanda da ESF. Para arquitetar o plano, foi necessário realizar um primeiro momento com avaliação, contendo vários modos de avaliar e examinar próprios de cada profissão. Após essa etapa de avaliação, a equipe traçava um plano individual para cada usuário, visando atendê-los interprofissionalmente e melhorar a condição funcional do idoso domiciliado (SILVA AL, et al., 2016)

Um aspecto importante observado durante a experiência foi a falta de apoio por parte dos familiares de grande parte dos idosos domiciliados. Tal situação afeta negativamente na qualidade de vida dos mesmos, uma vez que, de acordo com Aguiaro FF (2016), a família não possui apenas o papel reprodutivo devendo, também, ser a fonte de afeto, carinho e, principalmente, de respeito para com o idoso.

À medida que os indivíduos entram na fase do envelhecimento, muitas famílias os abandonam e passam a vê-los como impotentes, frágeis e indefesos, considerados muitas vezes "apenas mais um gasto e trabalho". Diante disto, muitos familiares acabam inserindo idosos em instituições de acolhimento, onde estes são afastados de suas rotinas de vida diária, assim como de seus parentes e amigos.

Além disso, outros fatores agravantes como condições precárias de moradia, presença de fatores de risco de quedas, limitações de locomoção para além dos limites domiciliares e negligência quanto a atividades básicas da vida diária, foram fatores marcantes durante as visitas. Perante a estes fatos, têm-se os princípios da Organização das Nações Unidas (ONU) sobre a pessoa idosa que são claramente descumpridos nestes casos, indo de contra aos direitos preestabelecidos. De acordo com o Relatório Azul (2017), estas pessoas têm direito a independência, acesso à alimentação, água, moradia, atenção à saúde adequada e devem ser de responsabilidade da família e da comunidade garantir e respeitar esses direitos.

As visitas domiciliares realizadas pela equipe interprofissional do PET-saúde apresentam potencialidades e limitações. No que tange às limitações, corroborando com os estudos de Moroco NFP (2014), elas encontram-se principalmente na sobrecarga de trabalho e na administração do tempo cronológico para a realização das visitas, em relação ao deslocamento até a residência do indivíduo e o tempo gasto durante a visita. Além disso, há o fato da disponibilidade do horário que as famílias têm para receber os profissionais, que muitas vezes é limitado, coincidindo com horários de almoço, dentre outros.

Concomitante a isso, tem-se a dificuldade da inserção da ótica do trabalho inter e multiprofissional no escopo da formação em saúde; essa inserção é trabalhada dentro do grupo do PET, porém, dificilmente com os estudantes que ficaram de fora do projeto.

A transformação da formação e das práticas em saúde, principalmente no que tange à Atenção Básica, é um desafio a ser superado, pois implica mudança de paradigmas já estruturados nos serviços, nas instituições de ensino e nas relações interpessoais. Com o diálogo e a aproximação, no âmbito das práticas e concepções atuais de atenção à saúde, pode diminuir desproporção entre a formação e realidade dos serviços. Dessa maneira, a construção de uma nova forma de trabalho em saúde centrada no usuário, com qualidade, resolubilidade e equidade podem ser favorecidas (NASCIMENTO DDG e OLIVEIRA CMA, 2010).

Em relação às potencialidades têm-se a avaliação dos idosos de acordo com aspectos biopsicossociais, ou seja, com atenção as demandas biológicas, psicológicas e sociais. Tal avaliação auxilia na identificação 
de possíveis demandas assistenciais, sendo realizada em ambiente domiciliar, facilitando a acessibilidade deste público ao atendimento, haja vista que a maioria possui dificuldade quanto ao deslocamento a unidade de saúde. Diante disso, de acordo com Oliveira SG, et al. (2018), as visitas domiciliares viabilizam a assistência à saúde por meio de ações de proteção e promoção, possibilitando diversos tipos de tratamento e garantindo o direito à saúde a todos os indivíduos, em conformidade com o artigo $196^{\circ}$ da constituição federal de 1988.

Além disso, o grupo que realizava as visitas era contemplado por discentes e preceptoras de áreas variadas, permitindo assim, uma visão conjunta e interprofissional, analisando aspectos essenciais para o trabalho envolvendo o idoso e todo o seu contexto. Diante disso, tal diversidade de olhares proporciona impactos positivos à saúde do idoso, uma vez que segundo Arruda LS e Moreira COF (2018), as práticas relacionadas a este público requerem um olhar abrangente, no qual cada profissional/estudante seja capaz de identificar as limitações quanto ao seu espaço de atuação, fomentando a prática colaborativa entre os diversos profissionais, a fim de possibilitar uma assistência mais completa e integral a todos os indivíduos.

Diante da experiência, observou-se que as visitas domiciliares, portanto, apresentam mais potencialidades do que limitações, uma vez que a mesma cumpre seu papel na saúde da comunidade à medida que responde aos princípios básicos da Atenção Básica e do SUS. Portanto, a experiência relatada mostra efeitos positivos que a educação interprofissional pode complementar na formação acadêmica dos futuros profissionais de saúde, e em específico desenvolver qualidades de uma assistência integral aos usuários do SUS, nesse contexto, o público idoso.

Por meio do levantamento dos dados atribuídos a pesquisa realizada, concluiu-se que, as visitas domiciliares, além se apresentarem como uma das principais estratégias para o acompanhamento da saúde, situação sanitária e qualidade de vida do idoso, contribuem para a formação de novos profissionais da área, sobretudo no desenvolvimento de competências éticas voltadas para o atendimento humanizado.

\section{AGRADECIMENTOS E FINANCIAMENTO}

Agradecemos a Universidade do Estado do Pará (UEPA) e a Secretaria Municipal de Saúde de Belém por proporcionar a inserção dos estudantes nos cenários da atenção básica, proporcionando a aquisição de uma gama de conhecimentos e trabalho interprofissional. Agradecemos também a equipe de profissionais da Unidade Municipal de Saúde pelo acompanhamento e articulação das visitas domiciliares. O Financiamento do trabalho foi realizado pelos próprios autores.

\section{REFERÊNCIAS}

1. AGUIARO FF. O IDOSO COMO CIDADÃO: Enfrentando o abandono familiar da pessoa idosa. Monografia (Bacharel em Serviço Social) - Universidade Federal Fluminense, Rio de Janeiro, 2016; $57 \mathrm{p}$.

2. ANDRADE, VMP; CARDOSO, CL. Visitas domiciliares de agentes comunitários de saúde: concepções de profissionais e usuários. Psico-USF, 2017; 22(1): 87-98.

3. ARRUDA LS, MOREIRA COF. Colaboração interprofissional: um estudo de caso sobre os profissionais do Núcleo de Atenção ao Idoso da Universidade Estadual do Rio de Janeiro (NAI/UERJ), Brasil. Interface-Comunicação, Saúde, Educação, 2018; 22(64):199-210.

4. ASSEMBLEIA LEGISLATIVA DO RIO GRANDE DO SUL. Relatório Azul: Garantias e Violação dos Direitos Humanos. Comissão de Cidadania e Direitos Humanos, $2017 . \quad$ Disponível em: http://www.al.rs.gov.br/FileRepository/repdcp_m505/CCDH/Relat\%C3\%B3rio\%20Azul\%202017.pdf. Acesso em: 5 fev. 2020

5. BRASIL. Ministério da Saúde. Programa de Educação pelo Trabalho para a Saúde (PET-Saúde). Brasília, 2020. Disponível em: <https://www.saude.gov.br/trabalho-educacao-e-qualificacao/gestao-da-educacao/qualificacaoprofissional/44938-programa-de-educacao-pelo-trabalho-para-a-saude-pet-saude>. Acesso em: 04 fev. 2020.

6. BRASIL. Portaria nำ 2.488, de 21 de outubro de 2011. Aprova a Política Nacional de Atenção Básica, estabelecendo a revisão de diretrizes e normas para a organização da Atenção Básica, para a Estratégia Saúde da Família (ESF) e o Programa de Agentes Comunitários de Saúde (PACS). Diário Oficial da União, n. 204, 2011.

7. BRASIL. Presidência da República. LEI No 10.741, DE 1ํ DE OUTUBRO DE 2003. Dispõe sobre o Estatuto do idoso. 2007. Disponível em: http://www.planalto.gov.br/ccivil_03/leis/2003/l10.741.htm. Acesso em 10 fev. 2020

8. CASTRO APR, de et al. Promoção da saúde da pessoa idosa: ações realizadas na atenção primária à saúde. Revista Brasileira de Geriatria e Gerontologia, 2018; 21(2): 155-163.

9. IBGE. Instituto Brasileiro de Geografia e Estatística. Projeção da população do Brasil e das Unidades da Federação. Disponível em: https://www.ibge.gov.br/apps//populacao/projecao/. Acesso em 03 maio 2020. 
10. LOURENÇO RA, VERAS RP. Mini Exame do Estado Mental: características psicométricas em idosos ambulatoriais. Revista de Saúde Pública, 2006; 40(4):712-719.

11. MELO DM, BARBOSA A.JG. O uso do Mini-Exame do Estado Mental em pesquisas com idosos no Brasil: uma revisão sistemática. Ciência \& Saúde Coletiva, 2015; 20(12):3865-3876.

12. MIRANDA GMD, et al. O envelhecimento populacional brasileiro: desafios e consequências sociais atuais e futuras, 2016; 19(3):507-519.

13. MOROCO NFP. Limitações e potencialidades da visita domiciliar como ferramenta assistencial na saúde da família. Monografia (Especialização em Atenção Básica em Saúde da Família) -Universidade Federal de Minas Gerais, Belo Horizonte, 2014; 25 p.

14. NASCIMENTO DDG, OLIVEIRA CMA. Reflexões sobre as competências profissionais para o processo de trabalho nos Núcleos de Apoio à Saúde da Família. O Mundo da Saúde, 2010; 34(1): 92-96.

15. OLIVEIRA SG, et al. Visita domiciliar no Sistema Único de Saúde. Revista Uruguaya de Enfermería, 2018; 13(1): 921.

16. PARADELA EMP, et al. Validação da escala de depressão geriátrica em um ambulatório geral. Revista de Saúde Pública, 2005; 39(6): 918-923.

17. REIP. Rede Regional de Educação Interprofissional da América. O que é educação interprofissional. 2018. Disponível em: <https://www.educacioninterprofesional.org/pt/o-que-e-educacao-interprofissional>. Acesso em: 04 fev. 2020.

18. SILVA AI, et al. Projeto terapêutico singular para profissionais da estratégia de saúde da família. Cogitare Enfermagem, $2016 ; 21(3): 1-8$. 